\title{
Long-term outcome of elderly patients with severe aortic stenosis as a function of treatment modality
}

\author{
Thomas Pilgrim, ${ }^{1}$ Lars Englberger, ${ }^{2}$ Martina Rothenbühler, ${ }^{3}$ Stefan Stortecky, \\ Osman Ceylan, ${ }^{1}$ Crochan J O'Sullivan, ${ }^{1}$ Christoph Huber, ${ }^{2}$ Fabien Praz, ${ }^{1}$ \\ Lutz Buellesfeld, ${ }^{1}$ Bettina Langhammer, ${ }^{2}$ Bernhard Meier, ${ }^{1}$ Peter Jüni, ${ }^{3}$ \\ Thierry Carrel, ${ }^{2}$ Stephan Windecker, ${ }^{1}$ Peter Wenaweser ${ }^{1}$
}

\begin{abstract}
- Additional material is published online only. To view please visit the journal online (http://dx.doi.org/10.1136/ heartjnl-2014-306106).

'Department of Cardiology, Bern University Hospital, Bern, Switzerland

${ }^{2}$ Department of Cardiovascular Surgery, Swiss Cardiovascular Center Bern, Bern University Hospital, Bern, Switzerland ${ }^{3}$ Institute of Social and Preventive Medicine and Clinical Trials Unit, Bern University Hospital, Bern, Switzerland
\end{abstract}

\section{Correspondence to} Dr Peter Wenaweser, Department of Cardiology, Swiss Cardiovascular Center Bern, Bern University Hospital, CH-3010 Bern, Switzerland; peter.wenaweser@insel.ch

Received 28 April 2014 Revised 4 August 2014 Accepted 5 August 2014 Published Online First 27 August 2014

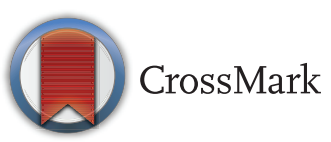

To cite: Pilgrim T,

Englberger L,

Rothenbühler $\mathrm{M}$, et al. Heart 2015;101:30-36.

\section{ABSTRACT}

Objective To assess long-term clinical outcomes of consecutive high-risk patients with severe aortic stenosis according to treatment allocation to transcatheter aortic valve implantation (TAVI), surgical aortic valve replacement (SAVR) or medical treatment (MT). Methods Patients with severe aortic stenosis were consecutively enrolled into a prospective single centre registry.

Results Among 442 patients (median age 83 years, median STS-score 4.7) allocated to MT ( $n=78)$, SAVR $(n=107)$, or TAVI $(n=257)$ all-cause mortality amounted to $81 \%, 37 \%$ and $43 \%$ after a median duration of follow-up of 3.9 years $(p<0.001)$. Rates of major adverse cerebro-cardiovascular events were lower in patients undergoing SAVR or TAVI as compared with MT (SAVR vs MT: HR $0.31,95 \% \mathrm{Cl} 0.21$ to 0.46 ) (TAVI vs MT: HR $0.34,95 \%$ Cl 0.25 to 0.46$)$, with no significant difference between SAVR and TAVI (HR $0.88,95 \% \mathrm{Cl}$ 0.62 to 1.25 ). Whereas SAVR (HR $0.39,95 \% \mathrm{Cl} 0.24$ to 0.61 ), TAVI (HR $0.37,95 \% \mathrm{Cl} 0.26$ to 0.52 ), and female gender (HR $0.72,95 \% \mathrm{Cl} 0.53$ to 0.99 ) were associated with improved survival, body mass index $\leq 20 \mathrm{~kg} / \mathrm{m}^{2}$ (HR $1.60,95 \% \mathrm{Cl} 1.04$ to 2.47 ), diabetes (HR $1.48,95 \% \mathrm{Cl}$ 1.03 to 2.12 ), peripheral vascular disease (HR 2.01, $95 \% \mathrm{Cl} 1.44$ to 2.81), atrial fibrillation (HR 1.74, 95\% $\mathrm{Cl} 1.28$ to 2.37 ) and pulmonary hypertension (HR 1.43, $95 \% \mathrm{Cl} 1.03$ to 2.00 ) were identified as independent predictors of mortality.

Conclusions Among high-risk patients with severe aortic stenosis, long-term clinical outcome through 5 years was comparable between patients allocated to SAVR or TAVI. In contrast, patients with MT had a dismal prognosis.

\section{INTRODUCTION}

Transcatheter aortic valve implantation (TAVI) has evolved into a valuable treatment option among elderly patients with symptomatic valvular aortic stenosis at high surgical risk. Evidence from randomised clinical trials showed a sustained survival benefit of TAVI compared with medical treatment (MT) in inoperable patients as well as similar survival as surgical aortic valve replacement (SAVR) among patients at high risk for surgery through 2 years of follow-up. ${ }^{12}$ However, data on longterm clinical outcomes of patients undergoing TAVI remain sparse and the rate of adverse events is largely determined by comorbidities. Long-term efficacy data of TAVI compared with surgical treatment are important to further refine the clinical selection process of TAVI candidates and to supplement in vitro data on valve durability.

We have previously reported the outcome of unselected patients allocated to a particular treatment strategy (MT, SAVR or TAVI) and presented periprocedural and midterm clinical outcome. ${ }^{3}$ The aim of the present analysis was to extend these observations during long-term follow-up through 5 years according to initial treatment allocation and to identify predictors of adverse clinical outcomes.

\section{METHODS \\ Patient population}

Between July 2007 and September 2010, all patients with symptomatic, severe aortic stenosis deemed at increased surgical risk were consecutively enrolled into a prospective single centre registry. Criteria for inclusion have been reported previously ${ }^{3}$ and are summarised in the online supplementary material. All subjects provided written informed consent and the study was approved by the local ethical committee.

\section{Interdisciplinary evaluation and selection of a treatment strategy}

Patients with severe aortic stenosis at increased surgical risk underwent a multimodal assessment according to a standardised protocol during a short hospitalisation (see online supplementary material). Selection of an appropriate treatment strategy was based on a consensus decision within the Heart Team.

MT consisted of treatment of cardiac and noncardiac comorbidities according to best clinical practice. In patients with severe coronary artery disease and limiting angina, percutaneous coronary intervention was performed as indicated. Balloon aortic valvuloplasty was not offered as part of the MT strategy, nor as a bridge to SAVR in patients with congestive heart failure. In some cases, treatment allocation was reconsidered and discussed in the Heart Team. As a consequence some patients underwent TAVI or SAVR at a later stage.

SAVR was performed according to contemporary standards as outlined previously. ${ }^{3}$

TAVI was performed with either the Medtronic CoreValve Revalving system or the Edwards SAPIEN valve through a transfemoral, transapical 
or a trans-subclavian access using standard techniques. The algorithm for access and device selection according to annulus diameter and vascular access dimensions has been reported previously. ${ }^{3}$

\section{Data collection}

Patients were included into the registry at the time of SAVR or TAVI; for patients undergoing MT the date of hospitalisation for multimodal evaluation was determined as the date of inclusion. Follow-up was performed by means of standardised telephone interviews or clinical visits. Between March and October 2013 we performed a sweep follow-up for all patients. Medical records, discharge summaries and documentation of hospitalisation were systematically collected from referring hospitals and general practitioners. Patients and procedure characteristics as well as follow-up data were entered into a dedicated database held at the Clinical Trials Unit in Bern, Switzerland.

\section{Definitions}

The definitions applied for the analysis have been reported in detail previously ${ }^{3}$ and are summarised in the online supplementary material. All events were adjudicated by a team of interventional cardiologists and cardiac surgeons.

\section{Statistical analysis}

Continuous variables are presented as mean \pm SD if their distribution is approximately normal and as median/range otherwise. The means were compared using analysis of variance and the differences in medians were evaluated with Mann-Whitney or Kruskal-Wallis tests. Categorical data are expressed as frequency (percentages), and were compared using $\chi^{2}$ and Fisher's exact tests. Survival was estimated using the Kaplan-Meier method and differences in estimates were compared by means of the log-rank test. All time-to-event analyses were based on the initial treatment allocation, in analogy to the intention-to-treat principle unless otherwise specified. The at-risk time span was derived from the date of intervention on one side and the last available date of a patient on the other side, determined either by the date of death, of the last follow-up or information coming from referring hospitals and practitioners. Univariate and multivariate Cox proportional hazards models were used to derive crude and adjusted survival estimates and to assess the association of baseline characteristics with clinical outcomes. All $\mathrm{p}$ values and 95\% CIs are two-sided and all analyses were performed using STATA release V.13.

\section{RESULTS}

Between April 2007 and September 2010, 452 patients with severe aortic stenosis at increased risk for surgery were assessed by the Heart Team. Ten patients died before definitive treatment allocation, 78 patients were assigned to MT, SAVR was performed in 107 patients and 257 patients underwent TAVI (see online supplementary figure S1). Patient characteristics at the time of the intervention are summarised in table 1 and online supplementary table S1. Whereas patients in the MT and the TAVI arms were comparable in terms of baseline characteristics

Table 1 Baseline characteristics

\begin{tabular}{|c|c|c|c|c|c|}
\hline & $\begin{array}{l}\text { Overall } \\
n=442\end{array}$ & $\begin{array}{l}\text { MT } \\
n=78\end{array}$ & $\begin{array}{l}\text { SAVR } \\
n=107\end{array}$ & $\begin{array}{l}\text { TAVI } \\
n=257\end{array}$ & p Value \\
\hline Age: median (IQR) & $83(8)$ & $84(7)$ & $80(8)$ & $83(7)$ & $<0.001$ \\
\hline Women & $230(52.0 \%)$ & $33(42.3 \%)$ & $53(49.5 \%)$ & $144(56.0 \%)$ & 0.090 \\
\hline BMI $\left(\mathrm{kg} / \mathrm{m}^{2}\right)$ : mean (SD) & $25.6(4.8)$ & $24.4(3.5)$ & $26.0(4.7)$ & $25.8(5.1)$ & 0.030 \\
\hline \multicolumn{6}{|l|}{ Cardiac risk factors } \\
\hline Hypertension & $338(76.5 \%)$ & $52(66.7 \%)$ & $85(79.4 \%)$ & $201(78.2 \%)$ & 0.080 \\
\hline Current smoker & $65(14.7 \%)$ & $7(9.0 \%)$ & $16(15.0 \%)$ & $42(16.3 \%)$ & 0.270 \\
\hline Diabetes mellitus & $101(22.9 \%)$ & $18(23.1 \%)$ & $21(19.6 \%)$ & $62(24.1 \%)$ & 0.650 \\
\hline Hypercholesterolaemia & $233(52.7 \%)$ & $32(41.0 \%)$ & $46(43.0 \%)$ & $155(60.3 \%)$ & 0.001 \\
\hline \multicolumn{6}{|l|}{ Past medical history } \\
\hline Prior MI & 79 (17.9\%) & $23(29.5 \%)$ & $9(8.4 \%)$ & $47(18.3 \%)$ & 0.001 \\
\hline Prior $\mathrm{PCl}$ & $77(17.4 \%)$ & $10(12.8 \%)$ & $9(8.4 \%)$ & $58(22.6 \%)$ & 0.003 \\
\hline CABG & $76(17.2 \%)$ & $18(23.1 \%)$ & $4(3.7 \%)$ & $54(21.0 \%)$ & $<0.001$ \\
\hline Stroke & $44(10.0 \%)$ & $13(16.7 \%)$ & $8(7.5 \%)$ & $23(9.0 \%)$ & 0.080 \\
\hline PVD & $93(21.0 \%)$ & $16(20.5 \%)$ & $13(12.1 \%)$ & $64(24.9 \%)$ & 0.030 \\
\hline \multicolumn{6}{|l|}{ Symptoms } \\
\hline NYHA III or IV & $251(56.9 \%)$ & $48(62.3 \%)$ & $48(44.9 \%)$ & $155(60.3 \%)$ & 0.014 \\
\hline Angina & $157(35.5 \%)$ & $27(34.6 \%)$ & $55(51.4 \%)$ & $75(29.2 \%)$ & $<0.001$ \\
\hline Syncope & $51(11.5 \%)$ & $14(18 \%)$ & $13(12.1 \%)$ & $24(9.3 \%)$ & 0.110 \\
\hline Atrial fibrillation & $105(23.8 \%)$ & $20(25.6 \%)$ & $19(17.8 \%)$ & $66(25.7 \%)$ & 0.250 \\
\hline \multicolumn{6}{|l|}{ Risk assessment } \\
\hline Log. EuroSCORE: median (IQR) & $19.1(19.9)$ & $25.8(22.5)$ & $9.8(8.2)$ & $22.4(19.9)$ & $<0.001$ \\
\hline Lin. EuroSCORE: median (IQR) & $10(4)$ & $11(2)$ & $8(2)$ & $11(3)$ & $<0.001$ \\
\hline STS score: median (IQR) & $4.7(3.6)$ & $5.4(4.7)$ & $3.4(2.7)$ & $5.1(3.8)$ & $<0.001$ \\
\hline \multicolumn{6}{|l|}{ Medications } \\
\hline Acetylsalicylic acid & $254(57.5 \%)$ & $39(50.0 \%)$ & $59(55.1 \%)$ & $156(60.7 \%)$ & 0.210 \\
\hline Clopidogrel & $62(14.0 \%)$ & $10(12.8 \%)$ & $5(4.7 \%)$ & $47(18.3 \%)$ & 0.003 \\
\hline Oral anticoagulation & $114(25.8 \%)$ & $22(28.2 \%)$ & $18(16.8 \%)$ & $74(28.8 \%)$ & 0.050 \\
\hline
\end{tabular}


Table 2 Long-term clinical outcome

\begin{tabular}{|c|c|c|c|c|c|}
\hline & $\begin{array}{l}\text { Overall } \\
\mathrm{n}=442\end{array}$ & $\begin{array}{l}\text { MT } \\
\mathrm{n}=78\end{array}$ & $\begin{array}{l}\text { SAVR } \\
\mathrm{n}=107\end{array}$ & $\begin{array}{l}\text { TAVI } \\
\mathrm{n}=257\end{array}$ & $\mathbf{P}_{\text {diff. between groups }}$ \\
\hline \multicolumn{6}{|l|}{ At 3 years } \\
\hline All-cause death & $182(41.2 \%)$ & $55(70.5 \%)$ & $33(30.8 \%)$ & $94(36.6 \%)$ & $<0.001$ \\
\hline Cardiovascular death & $132(29.9 \%)$ & $52(66.7 \%)$ & $18(16.8 \%)$ & $62(24.1 \%)$ & $<0.001$ \\
\hline MI & $7(1.6 \%)$ & $2(2.6 \%)$ & $0(0.0 \%)$ & $5(2.0 \%)$ & 0.266 \\
\hline Major stroke & $23(5.2 \%)$ & $3(3.9 \%)$ & $8(7.5 \%)$ & $12(4.7 \%)$ & 0.463 \\
\hline Minor stroke/TIA & $5(1.1 \%)$ & $0(0.0 \%)$ & $3(2.8 \%)$ & $2(0.8 \%)$ & 0.167 \\
\hline All-cause death or major stroke & $189(42.8 \%)$ & $56(71.8 \%)$ & $36(33.6 \%)$ & $97(37.7 \%)$ & $<0.001$ \\
\hline All-cause death, major stroke or MI & $192(43.3 \%)$ & $57(73.1 \%)$ & $36(33.6 \%)$ & 99 (38.5\%) & $<0.001$ \\
\hline \multicolumn{6}{|l|}{ At 5 years } \\
\hline All-cause death & $213(48.2 \%)$ & $63(80.8 \%)$ & $39(36.5 \%)$ & $111(43.2 \%)$ & $<0.001$ \\
\hline Cardiovascular death & $157(35.5 \%)$ & $60(76.9 \%)$ & $24(22.4 \%)$ & $73(28.4 \%)$ & $<0.001$ \\
\hline MI & $10(2.3 \%)$ & $2(2.6 \%)$ & $0(0.0 \%)$ & $8(3.1 \%)$ & 0.169 \\
\hline Major stroke & $25(5.7 \%)$ & $3(3.9 \%)$ & $9(8.4 \%)$ & $13(5.1 \%)$ & 0.379 \\
\hline Minor stroke/TIA & $6(1.4 \%)$ & $0(0.0 \%)$ & $3(2.8 \%)$ & $3(1.2 \%)$ & 0.316 \\
\hline All-cause death or major stroke & $221(50.0 \%)$ & $64(82.1 \%)$ & $42(39.3 \%)$ & $115(44.8 \%)$ & $<0.001$ \\
\hline All-cause death, major stroke or MI & $227(51.4 \%)$ & $65(83.3 \%)$ & $42(39.3 \%)$ & $120(46.7 \%)$ & $<0.001$ \\
\hline
\end{tabular}

and estimated periprocedural risk, patients allocated to SAVR tended to be younger, had less comorbidities and lower calculated risk.

\section{Clinical outcome}

Periprocedural outcome has been reported previously. All comparisons between the three treatment arms are descriptive. The median duration of follow-up of patients undergoing MT, SAVR or TAVI amounted to 4.6 (range 3.4-6.5) years, 3.8 (range 2.86.1) years and 3.9 (range 2.6-5.8) years, respectively. One patient was lost to follow-up 2.8 years after SAVR. Event rates at 3 years and 5 years are summarised in table 2 . There was a statistically significant association between the mortality rate and the type of treatment after 3 years and 5 years. Rates of myocardial infarction and cerebrovascular events were low without significant differences between groups. All-cause mortality through 5 years amounted to $81 \%, 37 \%$ and $43 \%$, among patients allocated to MT, SAVR and TAVI, respectively $(p<0.001$; figure 1$)$.

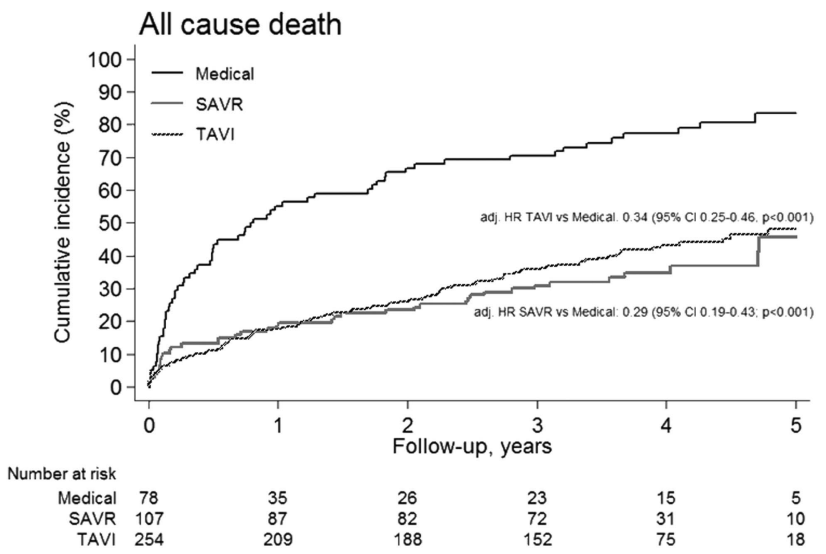

Figure 1 Cumulative incidence of all-cause mortality according to treatment strategy up to 5 years of follow-up. Medical treatment (black line), surgical aortic valve replacement (SAVR) (red line), transcatheter aortic valve implantation (TAVI) (blue line).
Crude and adjusted HRs for comparisons between groups are provided in online supplementary table S2. At 5 years of follow-up, the risk of the composite of all-cause death, major stroke and myocardial infarction was significantly lower for patients in the SAVR and TAVI groups as compared with the MT group in crude and adjusted analyses (SAVR vs MT: adj HR 0.31, 95\% CI 0.21 to 0.46 ; TAVI vs MT: adj HR $0.34,95 \%$ CI 0.25 to 0.46 ; figure 2). The difference was driven by all-cause mortality. No statistically significant difference in the risk of all-cause death, stroke and major myocardial infarction was observed between patients treated with SAVR or TAVI (SAVR vs TAVI: adj HR 0.88, $95 \%$ CI 0.62 to 1.25 ). A stratified comparison among major subgroups (age $\geq 80$ years, gender, STS-score $\geq 5$ and LVEF $<40 \%$ ) between patients undergoing intervention (SAVR or TAVI) versus MT demonstrated a consistent benefit for patients allocated to

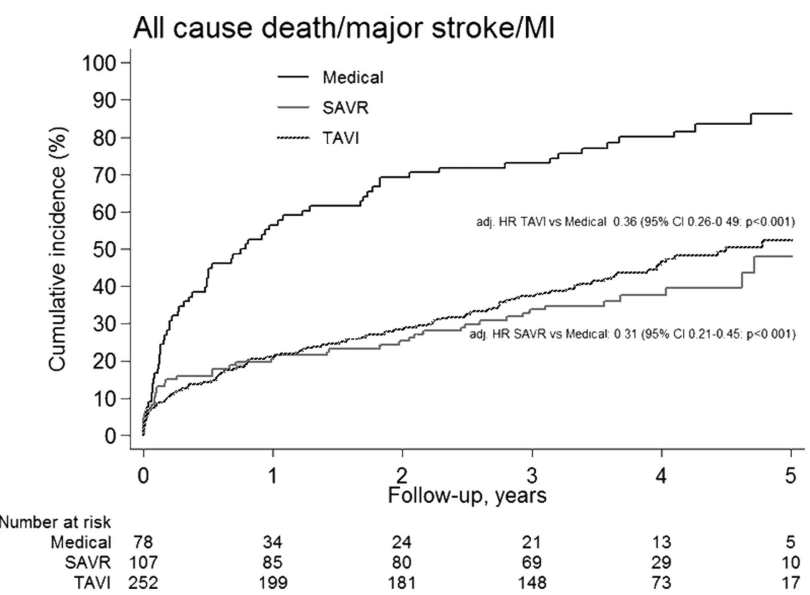

Figure 2 Cumulative incidence of major adverse cerebro-cardiovascular events defined as the combined end point of all-cause mortality, major stroke or myocardial infarction (MI) according to treatment strategy up to 5 years of follow-up. Medical treatment (black line), surgical aortic valve replacement (SAVR) (red line), transcatheter aortic valve implantation (TAVI) (blue line). 
Figure 3 Stratified analysis for all-cause mortality in major subgroups undergoing intervention (surgical aortic valve replacement (SAVR) or transcatheter aortic valve implantation $(\mathrm{TAVI})$ ) versus patients undergoing medical treatment. Black squares represent HRs; horizontal black lines illustrate Cls. A HR $>1$ (right side) is in favour of medical treatment, whereas a $H R<1$ (left side) is in favour of intervention (SAVR or TAVI). STS, Society of Thoracic Surgeons.

\begin{tabular}{|c|c|c|c|c|c|c|c|}
\hline & \multicolumn{7}{|c|}{ Stratified analysis for mortality at 5 years } \\
\hline & $\begin{array}{c}\text { SAVR/TAVI n } \\
(\%)\end{array}$ & $\begin{array}{c}\text { Medical } \\
\mathrm{n}(\%)\end{array}$ & $\mathrm{HR}(95 \% \mathrm{Cl})$ & & & p-value & $\begin{array}{c}\mathrm{p} \text {-value } \\
\text { interaction }\end{array}$ \\
\hline Overall & $150(41.2)$ & $63(80.8)$ & $0.32(0.24-0.44)$ & $\mapsto$ & & $<0.001$ & \\
\hline Age & & & & & & & 0.268 \\
\hline$<80$ years & $38(33.6)$ & $14(82.3)$ & $0.23(0.13-0.44)$ & $\longmapsto$ & & $<0.001$ & \\
\hline$\geq 80$ years & $112(44.6)$ & $49(80.3)$ & $0.37(0.26-0.52)$ & $\longmapsto$ & & $<0.001$ & \\
\hline Gender & & & & & & & 0.083 \\
\hline Male & $73(43.7)$ & $42(93.3)$ & $0.25(0.17-0.37)$ & $\longmapsto$ & & $<0.001$ & \\
\hline Female & $77(39.1)$ & $21(63.6)$ & $0.45(0.28-0.74)$ & $\longmapsto$ & & 0.001 & \\
\hline STS score & & & & & & & 0.2 \\
\hline$<5$ & $67(32.8)$ & $21(65.6)$ & $0.41(0.25-0.67)$ & $\longmapsto$ & & $<0.001$ & \\
\hline$\geq 5$ & $83(51.9)$ & $41(91.1)$ & $0.29(0.20-0.43)$ & $\longmapsto$ & & $<0.001$ & \\
\hline LVEF & & & & & & & 0.227 \\
\hline$\geq 40 \%$ & $113(40.9)$ & $32(78.1)$ & $0.39(0.26-0.58)$ & $\longmapsto$ & & $<0.001$ & \\
\hline \multirow[t]{2}{*}{$<40 \%$} & $29(48.3)$ & $27(90)$ & $0.28(0.16-0.48)$ & $\longmapsto$ & & $<0.001$ & \\
\hline & & & & $\begin{array}{lll}0.1 & 0.25 & 0.5\end{array}$ & 1 & 4 & \\
\hline
\end{tabular}

one of the two intervention arms (figure 3). After exclusion of the MT arm, a stratified analysis across subgroups between patients undergoing SAVR or TAVI identified no statistically significant differences between the two treatment strategies (see online supplementary figure S2).

\section{Cross-over from MT to SAVR or TAVI}

During the follow-up period, 11 of the 78 patients (14\%) initially assigned to MT were reassessed and crossed over to TAVI $(n=9)$ or SAVR $(n=2)$ after a median of 2.0 years (range 251159 days). Seven patients initially allocated to the MT group changed their mind and agreed to undergo TAVI $(n=6)$ or SAVR $(n=1)$. Four patients deemed poor candidates for intervention by the initial Heart Team consensus underwent cross-over to TAVI $(n=3)$ or SAVR $(n=1)$ after reassessment. There were no statistically significant differences in the baseline characteristics reported in table 1 between patients with or without cross-over from MT to one of the intervention arms, except for the STS score, which was significantly higher among the patients who did not cross over $(p=0.029)$. The majority of long-term survivors in the MT arm had undergone cross-over during the course of the study. After 5 years, only $6 \%$ of the patients who did not undergo conversion were alive, whereas the survival rate was $100 \%$ for the ones who subsequently underwent SAVR and $67 \%$ for those who underwent TAVI. The association between death and conversion either to SAVR or TAVI was statistically significant $(\mathrm{p}<0.001)$. A survival analysis of the overall population as-treated is depicted in figure 4.

\section{Predictors of mortality}

In multivariate analyses, SAVR (HR $0.39,95 \%$ CI 0.24 to 0.61 , $\mathrm{p}<0.001)$, TAVI (HR 0.37, 95\% CI 0.26 to $0.52, \mathrm{p}<0.001)$ and female gender (HR 0.72 , 95\% CI 0.53 to $0.99, \mathrm{p}=0.042$ ) were associated with improved survival. Conversely, body mass index (BMI) $\leq 20 \mathrm{~kg} / \mathrm{m}^{2}$ (HR 1.60, 95\% CI 1.04 to $2.47, \mathrm{p}=0.032$ ), diabetes (HR 1.48, 95\% CI 1.03 to $2.12, \mathrm{p}=0.033$ ), peripheral vascular disease (HR 2.01, 95\% CI 1.44 to 2.81, p<0.001), atrial fibrillation (HR $1.74,95 \%$ CI 1.28 to $2.37, \mathrm{p}<0.001$ ) and severe pulmonary hypertension (HR 1.43, 95\% CI 1.03 to $2.00, \mathrm{p}=0.034$ ) were identified as independent predictors of allcause mortality through 5 years of follow-up (table 3). After exclusion of patients in the MT arm, a BMI $\leq 20 \mathrm{~kg} / \mathrm{m}^{2}$, a history of stroke, a history of peripheral vascular disease and atrial fibrillation were identified as predictors of long-term mortality (see online supplementary table S3).

\section{Echocardiographic follow-up and valve-related reinterventions}

Echocardiographic follow-up was performed after a median of 3.3 years (range 3-1936 days) and 1.6 years (range 2461651 days) in patients undergoing SAVR or TAVI, respectively, $(\mathrm{p}<0.001)$. Due to the differing time intervals, the results must be considered descriptive. The mean trans-prosthetic gradient among patients of the TAVI group was significantly lower compared with patients of the SAVR group (see online supplementary figure S3). No statistically significant difference between the two treatment arms was documented with respect to the aortic valve area (TAVI $1.7 \pm 0.7 \mathrm{~cm}^{2}$ vs SAVR $1.4 \pm 0.4 \mathrm{~cm}^{2}, \mathrm{p}=0.11$ ). Conversely, moderate or severe aortic regurgitation was more common among patients treated with TAVI as compared with SAVR $(14.3 \%$ vs $3.1 \%, \mathrm{p}<0.001)$.

Valve-related repeat interventions beyond the periprocedural period were performed in five patients $(1.9 \%)$ in the TAVI cohort. In contrast, none of the patients of the SAVR group required a valve-related repeat intervention $(p=0.327)$. Two

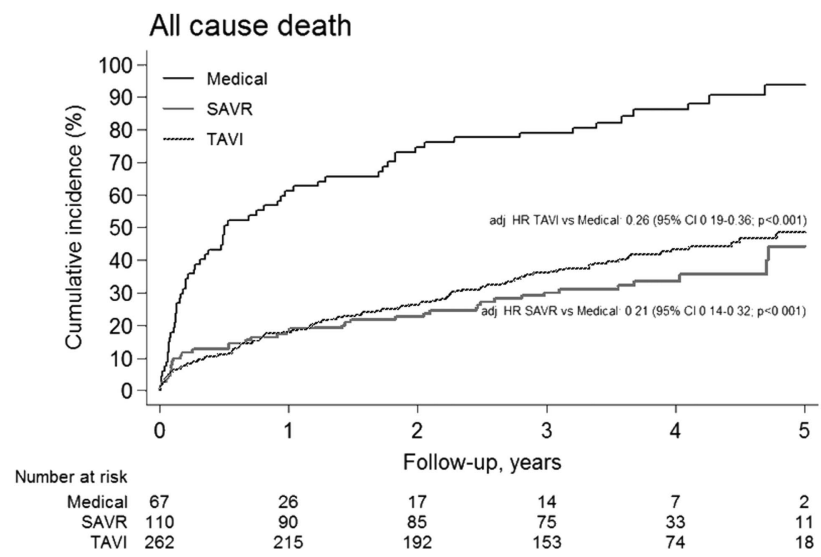

Figure 4 Cumulative incidence of all-cause mortality in an 'as treated' analysis up to 5 years of follow-up. Medical treatment (black line), surgical aortic valve replacement (SAVR) (red line), transcatheter aortic valve implantation (TAVI) (blue line). 
Table 3 Predictors of all-cause mortality at 5 years

\begin{tabular}{|c|c|c|c|c|c|c|}
\hline & Alive & Dead & Univariate HR $(95 \% \mathrm{CI})$ & p Value & Multivariate HR $(95 \% \mathrm{CI})$ & p Value \\
\hline Type of intervention & & & & $<0.001$ & & $<0.001$ \\
\hline Medical & $15(6.5 \%)$ & $63(29.6 \%)$ & Reference & & Reference & \\
\hline SAVR & $68(29.7 \%)$ & $39(18.3 \%)$ & $0.29(0.19-0.43)$ & & $0.39(0.24-0.61)$ & \\
\hline TAVI & $146(63.8 \%)$ & $111(52.1 \%)$ & $0.34(0.25-0.46)$ & & $0.37(0.26-0.52)$ & \\
\hline Age $\geq 80$ years & $151(65.9 \%)$ & $161(75.6 \%)$ & $1.38(1.01-1.89)$ & 0.045 & $1.39(0.98-1.97)$ & 0.067 \\
\hline Female & $132(57.6 \%)$ & $98(46.0 \%)$ & $0.70(0.53-0.92)$ & 0.010 & $0.72(0.53-0.99)$ & 0.042 \\
\hline $\mathrm{BMI} \leq 20 \mathrm{~kg} / \mathrm{m}^{2}$ & $18(7.9 \%)$ & $28(13.2 \%)$ & $1.42(0.94-2.15)$ & 0.091 & $1.60(1.04-2.47)$ & 0.032 \\
\hline Hypertension & $176(76.9 \%)$ & $162(76.1 \%)$ & $0.97(0.71-1.33)$ & 0.851 & - & \\
\hline Diabetes & $45(19.7 \%)$ & $56(26.3 \%)$ & $1.26(0.93-1.72)$ & 0.136 & $1.48(1.03-2.12)$ & 0.033 \\
\hline Hypercholesterolaemia & $123(53.7 \%)$ & $110(51.6 \%)$ & $0.90(0.69-1.18)$ & 0.463 & - & \\
\hline History of CHD & $132(57.6 \%)$ & $138(64.8 \%)$ & $1.23(0.93-1.65)$ & 0.139 & $1.13(0.82-1.58)$ & 0.453 \\
\hline History of MI & $34(14.9 \%)$ & $45(21.1 \%)$ & $1.33(0.95-1.85)$ & 0.095 & $0.81(0.55-1.21)$ & 0.306 \\
\hline History of CABG & $34(14.9 \%)$ & $42(19.7 \%)$ & $1.27(0.90-1.78)$ & 0.170 & $0.87(0.57-1.34)$ & 0.529 \\
\hline History of stroke & $14(6.1 \%)$ & $30(14.1 \%)$ & $1.94(1.32-2.85)$ & 0.001 & $1.52(1.00-2.32)$ & 0.051 \\
\hline History of PVD & $31(13.5 \%)$ & $62(29.1 \%)$ & $1.90(1.41-2.56)$ & $<0.001$ & $2.01(1.44-2.81)$ & $<0.001$ \\
\hline History of atrial fibrillation & $35(15.3 \%)$ & $70(32.9 \%)$ & $1.95(1.46-2.60)$ & $<0.001$ & $1.74(1.28-2.37)$ & $<0.001$ \\
\hline LVEF $<40 \%$ & $34(16.5 \%)$ & $56(27.9 \%)$ & $1.71(1.26-2.33)$ & 0.001 & $1.25(0.89-1.76)$ & 0.202 \\
\hline Peak PA pressure $\geq 60 \mathrm{~mm} \mathrm{Hg}$ & $40(17.5 \%)$ & $55(25.8 \%)$ & $1.50(1.10-2.04)$ & 0.010 & $1.43(1.03-2.00)$ & 0.034 \\
\hline
\end{tabular}

Values given are $\mathrm{n}(\%)$ or $\mathrm{HR}(95 \% \mathrm{Cl})$. Only variables with a $\mathrm{p}$ value $<0.25$ in the univariate analysis were included in the multivariate model.

$\mathrm{BMI}$, body mass index; $\mathrm{CABG}$, coronary artery bypass grafting; CHD, coronary heart disease; MI, myocardial infarction; PA, pulmonary artery; PVD, peripheral vascular disease;

SAVR, surgical aortic valve replacement; TAVI, transcatheter aortic valve implantation.

patients underwent balloon dilatation of the transcatheter prosthesis due to paravalvular regurgitation 13 days and 14 days after TAVI. One patient was diagnosed with an aorto-right ventricular fistula 38 days after implantation of an Edwards SAPIEN prosthesis which was occluded with a coil. ${ }^{4}$ One patient developed severe paravalvular aortic regurgitation 1.3 years after implantation of a Medtronic CoreValve bioprosthesis and was treated with valve-in-valve implantation of a second Medtronic CoreValve bioprosthesis. And one patient developed severe valvular deterioration 4.6 years after implantation of an Edwards SAPIEN prosthesis (mean gradient $64 \mathrm{~mm} \mathrm{Hg}$, aortic valve area (AVA) $0.6 \mathrm{~cm}^{2}$ ) and was treated successfully by valve-in-valve- implantation (Medtronic CoreValve bioprosthesis).

\section{DISCUSSION}

We present clinical outcomes of elderly patients with severe symptomatic aortic stenosis at increased risk for surgery as a function of treatment modality through 5 years of follow-up. The key findings of our analysis can be summarised as follows: (1) TAVI and SAVR improve long-term survival compared with MT; (2) there were no statistically significant differences in rates of major adverse cerebro-cardiovascular events between patients allocated to SAVR and TAVI through 5 years of follow-up; (3) male gender, low BMI, diabetes, peripheral vascular disease, atrial fibrillation and severe pulmonary hypertension were identified as independent predictors of mortality at 5 years; (4) repeat interventions for prosthetic heart valve related problems were rare and were observed in TAVI patients only; (5) patients with cross-over from medical therapy to SAVR or transcatheter aorticvalve replacement (TAVR) had a reasonably good outcome whereas those who did not had a dismal prognosis.

As reported previously, a considerable proportion of patients in the MT arm refused intervention despite a recommendation by the Heart Team. ${ }^{3}$ TAVI and SAVR decreased the absolute risk of mortality through 5 years of follow-up by approximately $70 \%$ with low rates of myocardial infarction or cerebrovascular events. Our findings are in line with the 2-year results of the randomised PARTNER B trial showing an absolute risk reduction of $42 \%$ and $43 \%$ in the first 2 years after randomisation among patients treated with TAVI compared with MT, respectively. $^{2}$ In our analysis, the time-to-event curves between patients who underwent SAVR or TAVI and those who underwent MT diverged until 2 years after treatment allocation and run approximately in parallel for the follow-up beyond 2 years. This finding persisted in an 'as-treated' analysis and was not attributable to the patients in the MT arm with cross-over to intervention.

Efficacy of TAVI or SAVR was also apparent among patients who subsequently crossed over to an intervention. Patients with cross-over from MT to TAVI or SAVR were comparable in terms of baseline characteristics to patients who continued with MT. However, mortality was significantly lower among patients who crossed over compared with those who continued MT. This finding highlights the fact that the process of decision-making can be challenging within the Heart Team and patients with only moderate symptoms may refuse active treatment owing to fear of complications or acceptable quality of life under MT alone.

Rates of adverse events showed no significant differences between patients allocated to SAVR or TAVI with respect to major adverse cerebro-cardiovascular events throughout 5 years of follow-up despite differences in baseline characteristics. This is in line with the randomised comparison of SAVR and TAVI in the PARTNER A trial without differences in all-cause mortality or stroke throughout 3 years of follow-up. ${ }^{5}$ Rates of myocardial infarction and stroke were low in all groups during this follow-up period.

When assessing predictors of all-cause mortality at 5 years in a multivariate analysis, male gender, low BMI, diabetes, peripheral vascular disease, atrial fibrillation and severe pulmonary hypertension emerged as independent predictors. Low BMI is one of the determinants of frailty that has previously been 
associated with adverse outcome after $\mathrm{TAVI}^{6}{ }^{7}$ and remains an important piece in the puzzle for further improvements in this patient population. An appropriate assessment of frailty followed by a dedicated intervention before TAVI or SAVR might therefore be an additional strategy to guide patient selection. Diabetes also emerged an independent predictor of mortality confirming the findings of previous studies. ${ }^{8}$ A subanalysis of the PARTNER trial reported the effect of diabetes on outcomes and hypothesised improved survival of patients undergoing TAVI compared with SAVR (18.0\% vs $27.4 \%$, HR 0.60, 95\% CI 0.36 to $0.99, \mathrm{p}=0.04) .{ }^{9}$ Peripheral vascular disease, but not coronary artery disease was identified as an independent predictor in the present analysis. We have documented in a larger cohort of patients undergoing TAVI an association of advanced coronary artery disease with an increased risk of cardiac mortality. ${ }^{10}$ This finding may be related to the fact that peripheral vascular disease is a marker of atherosclerotic disease burden in general. Furthermore, patients with peripheral vascular disease have an increased risk of cerebrovascular events but also elevated rates of vascular access site complications. Another unfavourable marker is atrial fibrillation which has been associated with adverse outcome in previous studies. ${ }^{11}$ Finally, severe pulmonary hypertension has previously been associated with an increased risk of mortality after TAVI. ${ }^{12}$ In patients with fixed pulmonary hypertension, the poor outcome may be related to right heart failure despite successful elimination of the increased afterload imposed by the stenotic valve.

Another important consideration is valve durability after TAVI and SAVR. The low incidence of repeat interventions due to prosthetic heart valve complications among TAVI patients is reassuring. Only two patients (1.9\%) in the present cohort experienced late valve degeneration which was successfully treated by valve-in-valve intervention. The limited echocardiographic evaluation, although not prospectively defined, showed sustained low transvalvular gradients. This finding is supported by a recent publication by Toggweiler et $a l^{13}$ reporting on favourable haemodynamic outcome of patients treated with TAVI through 5 years of follow-up. Mild valvular aortic regurgitation was documented in $7.1 \%$ of 88 patients, and 3 patients $(3.4 \%)$ were found to have moderate valvular aortic regurgitation or stenosis at 5 years of follow-up. ${ }^{13}$ Rates of mild or moderate valvular aortic regurgitation as assessed by echocardiography were higher among patients undergoing TAVI as compared with SAVR $(\mathrm{p}<0.001)$.

Our analysis has several limitations. First, the patients were referred from affiliated medical centres and allocated to the different treatment strategies in a non-randomised fashion. We present adjusted analyses to correct for the selection bias. However, latent factors may have been underappreciated in our analysis. Second, we report on a relatively small cohort of patients. In turn, follow-up was almost complete and reports on long-term outcome of patients undergoing TAVI are scarce. Third, not all patients underwent echocardiographic follow-up and the duration of follow-up was considerably longer among patients who underwent SAVR as compared with those who underwent TAVI. Furthermore, patients with pathological findings on previous examinations might have been summoned up more regularly than patients with normal valvular function.

In summary, the current analysis confirms comparable clinical outcomes of TAVI and SAVR during long-term follow-up in a selected patient population. No statistically significant difference in outcome apart from the higher rate of residual aortic regurgitation in disfavour of the TAVI population was found.
In contrast, MT was associated with a dismal prognosis unless patients underwent conversion of treatment to SAVR or TAVI at a later stage.

\section{Key messages}

\section{What is known on this subject?}

Evidence from randomised clinical trials showed a sustained survival benefit of transcatheter aortic valve implantation (TAVI) compared with medical treatment (MT) in inoperable patients as well as similar survival as surgical aortic valve replacement (SAVR) among patients at high risk for surgery through 2 years of follow-up.

\section{What might this study add?}

This study provides long-term outcome through 5 years of a consecutive unselected cohort of patients at increased risk allocated to either one of all currently available strategies for the treatment of severe aortic stenosis. The study shows similar rates of major adverse cerebro-cardiovascular events for TAVI and SAVR, and dismal prognosis for patients undergoing MT. The study identifies low body mass index, diabetes, peripheral vascular disease, atrial fibrillation and pulmonary hypertension as independent predictors of mortality.

\section{How might this impact on clinical practice?}

The study might improve the selection of adequate candidates for TAVI and SAVR.

Contributors TP: Study design, data collection, data analysis and writing. LE: Study design, data collection and writing. MR and $\mathrm{CH}$ : Data analysis and writing. SS: Data collection and analysis. OC, FP, LB and BL: Data collection. CJO: Study design and data collection. BM: Writing. PJ: Data analysis. TC and SW: Study design, data collection and writing. PW: Study design, data collection, data analysis and writing.

Funding PW: Proctoring and lecture fees from Edwards Lifesciences and Medtronic; Research grant to the institution (University of Berne) from Medtronic.

$\mathrm{CH}$ : Proctoring and lecture fees from Edwards Life Sciences and Symetis; consultancy for Medtronic. SW: Research grants to the institution from Biotronik and St Jude.

Patient consent Obtained.

Ethics approval Bern Ethics Committee.

Provenance and peer review Not commissioned; externally peer reviewed.

\section{REFERENCES}

1 Kodali SK, Williams MR, Smith CR, et al. Two-year outcomes after transcatheter or surgical aortic-valve replacement. N Engl J Med 2012;366:1686-95.

2 Makkar RR, Fontana GP, Jilaihawi $\mathrm{H}$, et al. Transcatheter aortic-valve replacement for inoperable severe aortic stenosis. N Engl J Med 2012; 366:1696-704.

3 Wenaweser P, Pilgrim T, Kadner A, et al. Clinical Outcomes of patients with severe aortic stenosis at increased surgical risk according to treatment modality. J Am Coll Cardiol 2011:58:2151-62

4 Pilgrim T, Meier B, Wenaweser P. Aorto-right ventricular fistula after transfemoral aortic valve implantation. J Invasive Cardiol 2010;22:E30-1.

5 Thourani VH. Three-year outcomes after transcatheter or surgical aortic valve replacement in high-risk patients with severe aortic stenosis. Presented at: American College of Cardiology Scientific Session/i2 Summit; March 11, 2013; San Francisco, CA, USA.

6 Wenaweser P, Pilgrim T, Kadner A, et al. Clinical outcome and predictors for adverse events after transcatheter aortic valve implantation with the use of different devices and access routes. Am Heart J 2011;108:195-201.

7 Schoenenberger AW, Stortecky S, Neumann S, et al. Predictors of functional decline in elderly patients undergoing transcatheter aortic valve implantation (TAVI). Eur Heart J 2013;34:684-92 


\section{Valvular heart disease}

8 Conrotto F, D'Ascenzo F, Giordana F, et al. Impact of diabetes mellitus on early and midterm outcomes after transcatheter aortic valve implantation (from a multicenter registry). Am J Cardiol 2014;113:529-34.

9 Lindman BR, Pibarot P, Arnold SV, et al. Transcatheter versus surgical aortic valve replacement in patients with diabetes and severe aortic stenosis at high risk for surgery: an analysis of the PARTNER trial. J Am Coll Cardiol 2014;63:1090-9.

10 Stefanini GG, Stortecky S, Cao D, et al. Coronary artery disease severity and aortic stenosis: clinical outcomes according to SYNTAX-score in patients undergoing transcatheter aortic valve implantation. Eur Heart J 2014. In press.
11 Stortecky S, Buellesfeld L, Wenaweser P, et al. Atrial fibrillation and aortic stenosis: impact on clinical outcomes among patients undergoing transcatheter aortic valve implantation. Circ Cardiovasc Interv 2013; 6:77-84.

12 Ben-Dor I, Goldstein SA, Pichard AD, et al. Clinical profile, prognostic implication, and response to treatment of pulmonary hypertension in patients with severe aortic stenosis. Am J Cardiol 2011;107:1046-51.

13 Toggweiler $\mathrm{S}$, Humphries $\mathrm{KH}$, Lee $\mathrm{M}$, et al. 5-year outcome after transcatheter aortic valve implantation. J Am Coll Cardiol 2013;61:413-19. 